\title{
Direction of catheter insertion and the incidence of paresthesia during continuous epidural anesthesia in the elderly patients
}

\author{
Jong-Hak Kim, Jun Seop Lee, and Dong Yeon Kim \\ Department of Anesthesiology and Pain Medicine, School of Medicine, Ewha Womans University, Seoul, Korea
}

Background: Continuous epidural anesthesia is useful for endoscopic urologic surgery, as mostly performed in the elderly patients. In such a case, it is necessary to obtain successful sacral anesthesia, and the insertion of epidural catheter in the caudad direction may be needed. However, continuous epidural catherization has been related to paresthesias. This study aimed to evaluate the effects of the direction of the catheter insertion on the incidence of paresthesias in the elderly patients.

Methods: Two hundred elderly patients scheduled for endoscopic urologic surgery were enrolled. The epidural catheter was inserted at L2-3, L3-4, and L4-5 using the Tuohy needle. In Group I ( $=100)$, the Tuohy needle with the bevel directed the cephalad during the catheter insertion. In Group II $(n=100)$, it directed the caudad. During the catheter insertion, an anesthesiologist evaluated the presence of paresthesias and the ease or difficulty during the catheter insertion.

Results: In Group I ( $n=97), 15.5 \%$ of the patients had paresthesias versus $18.4 \%$ in Group II ( $n=98)$, and there was no significant difference between the two groups. In paresthesia depending on the insertion site and the ease or difficulty during the catheter insertion, there were no significant differences between the two groups.

Conclusions: Our results concluded that the direction of epidural catheter insertion did not significantly influence the incidence of paresthesias in the elderly patients. (Korean J Anesthesiol 2013; 64: 443-447)

Key Words: Catheters, Epidural anesthesia, Geriatric, Paresthesia.

Received: August 16, 2012. Revised: 1st, October 4, 2012; 2nd, October 19, 2012. Accepted: October 30, 2012.

Corresponding author: Dong Yeon Kim, M.D., Department of Anesthesiology and Pain Medicine, School of Medicine, Ewha Womans University, 911-1, Mok-dong, Yangcheon-gu, Seoul 158-710, Korea. Tel: 82-2-2650-5558, Fax: 82-2-2655-2924, E-mail: kdyeon@ewha.ac.kr (c) This is an open-access article distributed under the terms of the Creative Commons Attribution Non-Commercial License (http:// creativecommons.org/licenses/by-nc/3.0/), which permits unrestricted non-commercial use, distribution, and reproduction in any medium, provided the original work is properly cited. 


\section{Introduction}

Continuous epidural anesthesia is a commonly used regional anesthetic method, employed in various surgeries and for obstetrical pain control. As the method has many advantages over the general anesthesia in urology patients undergoing transurethral resection, the majority of whom are the elderly, continuous epidural anesthesias is the preferred anesthesia method.

When the local anesthetic is administered into the lumbar epidural space, the local anesthetic distributes toward the cephalad rather than the caudad direction, and there is a possibility of insufficient anesthesia at the L5 and S1 nerve roots, as the segment is thicker than other segments [1]. Although a number of factors contribute to the extent of the nerve block in epidural anesthesia, some published reports state that it is better to insert the epidural catheter toward the caudad than the cephalad direction for effective sacral anesthesia [2]. As such the administration of local anesthesia through the epidural catheter inserted toward the caudad side may be the more effective anesthetic method in urological procedures such as transurethral resection.

Paresthesia may occur during the insertion of the epidural catheter in continuous epidural anesthesia. Paresthesia can increase discomfort in patients undergoing the procedure; it can also increase the risk of sudden movements, which leads to an increase in the risk of post-operational nerve-related complications or nerve damage. There is a possibility that the catheter may touch the parvertebral tissues and the directly exposed nerve root when the epidural catheter is inserted toward the caudad direction, and reports have stated that paresthesia may occur more frequently in this approach than when the insertion direction is toward the cephalad direction [3]. However, there is no report on whether a relationship exists between the insertion of the catheter toward the caudad direction in lumbar epidural anesthesia and the occurrence of paresthesia in the elderly patients.

In this study, the authors inserted an epidural catheter toward the cephalad or caudad direction in the elderly urology patients who were undergoing lumbar epidural anesthesia for transurethral resection, and compared the occurrence of paresthesia according to these two insertion approaches.

\section{Materials and Methods}

The study subjects were 200 American Society of Anesthesiologists Class I-II elderly patients aged over 60 years old, who had been scheduled for elective endoscopic urologic surgery under lumbar epidural anesthesia in our hospital. Informed consent was obtained from all patients, and the protocol of the study was approved by the Institutional Review Board at our institution. Patients were randomly divided into two groups, the epidural needle bevel was directed cephalad (Group I, n = 100 ) or caudad (Group II, $n=100$ ). Exclusion criteria included previous spinal surgery or clotting abnormalities.

After arriving at the operating room without any premedication, all patients received $10 \mathrm{ml} / \mathrm{kg}$ of lactated Ringer's solution. Electrocardiogram, pulse oximetry, heart rate, and non-invasive blood pressure were monitored. With the patient in the left lateral position, after local infiltration with $2 \%$ lidocaine at the L2-3, L3-4, or L4-5 interspace, lumbar epidural puncture was performed using a midline approach with $18 \mathrm{G}$ Tuohy needle and the loss of resistance technique. After the bevel of needle was directed cephalad or caudad, $20 \mathrm{G}$ epidural catheter (BD Perisafe $^{\mathrm{TM}}$, BD Medical System, Belgium) was inserted $3 \mathrm{~cm}$ into epidural space, and the test dose drug $(1.5 \%$ lidocaine $3 \mathrm{ml}$ with $1: 200,000$ epinephrine) was injected through the catheter to rule out vascular or subarachnoid injection of drugs. After the procedure, the patients were placed supine.

All the procedures were done by the same expert anesthesiologist, who judged the insertion of the catheter as easy or difficult (with resistance to insertion). During catheter insertion, another anesthesiologist who was unaware of the bevel direction evaluated the response of the patient and the presence of paresthesias, and recorded its site as 'at back' (restricted to back) or 'beyond back' (radiated to hip or leg). Sensory block was assessed with alcohol cotton at the interval of $1 \mathrm{~min}$ and $5 \mathrm{~min}$ after the test dose. When the sensory block occurred, the full $13 \mathrm{ml}$ of local anesthetic was injected into the epidural space in 3 divided doses ( $2 \%$ lidocaine $3 \mathrm{ml}, 2 \%$ lidocaine $5 \mathrm{ml}$, and $0.5 \%$ bupivacaine $5 \mathrm{ml}$ ) at the interval of $1 \mathrm{~min}$. At one day after surgery, an anesthesiologist visited the patients and confirmed the presence of neurologic complications at $24 \mathrm{hr}$ after the epidural anesthesia.

All the results are presented as mean \pm standard deviation or the number of patients. Statistical analysis was performed using SPSS (version 12.0, SPSS Inc., USA). Demographic data were compared using t-test; and the analysis of paresthesias used chi-square test or Fisher's exact test. A P value $<0.05$ was considered statistically significant.

\section{Results}

There were no significant differences in patient characteristics between the two groups (Table 1). Among the patients, 3 patients of Group I and 2 patients of Group II were excluded, because of the impossibility of catheter insertion during the epidural anesthesia.

In the presence of paresthesia, $15.5 \%$ of the patients in Group I ( $\mathrm{n}=97)$ had paresthesias versus $18.4 \%$ in Group II $(\mathrm{n}=98)$, and there was no significant difference between the two groups 
Table 1. Demographic Data

\begin{tabular}{lcc}
\hline & Group I $(\mathrm{n}=97)$ & Group II $(\mathrm{n}=98)$ \\
\hline Age $(\mathrm{yr})$ & $68.4 \pm 0.4$ & $66.7 \pm 0.5$ \\
Height $(\mathrm{cm})$ & $167.5 \pm 2.3$ & $166.1 \pm 2.0$ \\
Weight $(\mathrm{kg})$ & $61.9 \pm 1.3$ & $64.1 \pm 1.4$ \\
\hline
\end{tabular}

Values are presents as mean \pm SD. Group I: cephalad directed catheter. Group II: caudad directed catheter. There were no significant differences between the two groups.

Table 2. Incidence of Paresthesia during Insertion of the Epidural Catheter

\begin{tabular}{lcc}
\hline & Group I (n=97) & Group II (n=98) \\
\hline Paresthesia presence & $15(15.5)$ & $18(18.4)$ \\
At back & $3(3.1)$ & $4(4.1)$ \\
Beyond back & $12(12.4)$ & $14(14.3)$ \\
\hline
\end{tabular}

Values are presented as number of patients (\%). Group I: cephalad directed catheter. Group II: caudad directed catheter. There were no significant differences between the two groups.

(Table 2). In the sites of paresthesia, there was no significant difference between the two groups (Table 2). In paresthesia depending on the insertion site and the ease or difficulty during the catheter insertion, there were no significant differences between the two groups (Table 3).

All patients who were inserted epidural catheter gained good anesthesia for the surgery. There were no neurologic complications continuing at 24 hours after the epidural anesthesia in all patients, including the patients who complained of paresthesia.

\section{Discussion}

Continuous epidural anesthesia is a popular method of regional anesthesia, and at the present clinic, continuous epidural anesthesia is performed in the urological endoscopic surgery, such as transurethral resection, which is mainly performed in the elderly patients over 60 years of age. In these cases, sufficient nerve block is needed in the sacral area. Galindo et al. [1] reported that local anesthetic administered for lumbar epidural anesthesia distributes to a greater extent towards the cephalad direction; in addition, as the S1 nerve root is thicker, which may result in inadequate anesthesia in the sacral area, there is a need for a method that can assist the distribution of local anesthesia toward the sacral area. The study was initiated to insert the epidural catheter toward the caudad direction rather than the cephalad direction, which is the conventional practice. Chou et al. [2] compared the effects of catheter insertion in either the caudad or cephalad direction during the epidural anesthesia for the ankle surgery, considering the possibility of insufficient anesthesia at the S1 nerve root, with the thicker nerve root. It was reported that the group with
Table 3. Incidence of Paresthesia Depending on Insertion Site and Ease of Epidural Catheter

\begin{tabular}{lcc}
\hline & Group I (n=97) & Group II (n=98) \\
\hline A. Insertion site & & \\
L2-3 & 12 & 14 \\
L3-4 & 70 & 67 \\
L4-5 & 15 & 17 \\
Incidence of paresthesia & & \\
L2-3 & $2 / 12(16.7)$ & $3 / 14(21.4)$ \\
L3-4 & $10 / 70(14.3)$ & $12 / 67(17.9)$ \\
L4-5 & $3 / 15(20.0)$ & $3 / 17(17.6)$ \\
B. Ease of insertion & $93(95.9)$ & $93(94.9)$ \\
$\quad$ Easy & $4(4.1)$ & $5(5.1)$ \\
Difficult & & \\
Incidence of paresthesia & $13 / 93(14.0)$ & $15 / 93(16.1)$ \\
$\quad$ Easy & $2 / 4(50)$ & $3 / 5(60)$ \\
$\quad$ Difficult &
\end{tabular}

Values are presented as number of patients (\%). Group I: cephalad directed catheter. Group II: caudad directed catheter. There were no significant differences between the two groups.

insertion toward the caudad direction had a shorter time to reach anesthesia and had a better anesthetic effect.

Complications such as paresthesia and unintentional blood vessel or dural puncture may occur during the catheter insertion under continuous epidural anesthesia. The epidural space is positioned intraspinally, outside of the dura and surrounding the epidural sac and it encompasses the fat tissue, blood vessels, and nerve roots. Virtually no nerve distribution occurs in the posterior of dura; therefore, it is possible to carry out relatively safe continuous epidural anesthesia. However, if the catheter is moved toward the lateral side when the catheter is positioned in the epidural space, paresthesia can occur, as the catheter comes into contact with the spinal nerves, such as the nerve root or sinuvertebral nerve, which is distributed in the anterior epidural and around the nerve roots $[4,5]$. In addition, the catheter may puncture a blood vessel and become positioned within the blood vessel.

Paresthesia may occur during the insertion of the epidural catheter in continuous epidural anesthesia. The paresthesia can increase discomfort in patients undergoing the procedure, as well as increase the risk of sudden movements, which can be related to temporal or continuous nerve damage [6]. Nerverelated complications are more frequently reported in patients who experience paresthesia during regional anesthesia [6], and direct damage to the nerve root or spinal cord may be presented as paresthesia [7]. Reports have indicated the incidence of paresthesia during the epidural catheter insertion from $44 \%$ [8] to as high as $57 \%$ in pregnant mothers [9]. In addition, the prevalence of unintentional vascular puncture with radiologic confirmation has been reported at $1.4 \%$ [10]. In this study, the reported cases of paresthesia in the cephalad and caudad 
groups were $15.5 \%$ and $18.4 \%$, respectively. The figures were relatively lower than in other studies, which can be attributed to the study subjects being elderly patients who mainly complained of severe paresthesia only and did not respond sensitively to mild paresthesia.

A number of factors can affect paresthesia or vascular puncture. These factors may include the material of the catheter, epidural puncture being appropriately performed at the midline, and length of the catheter that proceeds toward the epidural space. When the epidural catheter is inserted away from the midline, it is easier to puncture the epidural blood vessel in the intervertebral foramen and increase the occurrence of paresthesia, compared with when it is inserted appropriately at the midline. There are studies with regard to reducing the occurrence of paresthesia. Cesur et al. [11] assessed the occurrence of paresthesia when the local anesthetic was administered prior to catheter insertion under epidural anesthesia. When the catheter was inserted using the conventional method, $31.6 \%$ experienced paresthesia, compared with $11 \%$ in the group that received administration of the local anesthetic prior to the catheter insertion. Cartagena and Gaiser [12] assessed parturients under continuous epidural anesthesia to control labor pain: $15.4 \%$ reported paresthesia when the conventional method was used, where the catheter was inserted $10 \mathrm{~cm}$ and pulled out $5 \mathrm{~cm}$; andparesthesia occurred in $7.5 \%$, when the catheter was directly inserted $5 \mathrm{~cm}$ only. Although no radiologic confirmation was done, the authors ensured that the epidural puncture was done correctly at the midline. When the bevel of the epidural needle was directed toward the cephalad or caudad direction, the bevel was positioned as close to the midline as possible, so that the catheter could be positioned in the midline rather than the lateral side.

Muñoz et al. [3] compared the occurrence of paresthesia in parturients under continuous epidural anesthesia for labor pain when the catheter was inserted either in the cephalad or caudad direction, and the occurrence of paresthesia in the cephalad and caudad groups were $20 \%$ and $40 \%$, respectively. Although twice as many patients in the caudad group reported paresthesia, no difference was reported in the degree of anesthesia, and none of the patients experienced neurological symptoms 24 hours after the anesthesia. Hence it was concluded that catheter insertion toward the caudad direction is not ideal. In addition, the occurrence of more frequent paresthesia can be regarded as resulting from the catheter insertion not being smooth through the caudad direction. The progression angle is narrower in the caudad direction than in the cephalad direction, where the progression angle is wider than a right degree angle. The slight difference in the rate of catheter progression in the two different directions may be the reason. The authors predicted a higher rate of paresthesia when the catheter is inserted toward the caudad rather than the cephalad direction, but the difference was not statistically significant. This can be attributed to the study sample being composed elderly patients with desensitized responses to paresthesia. The elderly patients complained of discomfort with severe paresthesia only, where the catheter was inserted laterally and in contact with the nerve. The chance of the epidural catheter inserted away from the midline and positioned laterally is the same in both the cephalad and caudad directions. Paresthesia localized in the back only can result from the stimulation of the sinuvertebral nerve; and the stimulation of the nerve root can be responsible for the effects radiating to the hip and leg. There were no significant differences between the cephalad and caudad direction.

Permanent nerve damage is rare despite the frequent occurrence of paresthesia under epidural catheter insertion [13]. Terasako [14] reported that as the patients under general anesthesia cannot respond to paresthesia during regional anesthesia, such method can increase the risks of postoperative neurological complications. However, the study results presented no neurological complications in 573 patients under general anesthesia with lumbar epidural anesthesia. Although there was no actual report of permanent nerve damage, the authors did not attempt further progression of the catheter, and there was no neurological complication that lasted longer than 24 hours.

This study was limited in that no radiologic confirmation was conducted for the appropriate positioning of the catheter toward the intended direction. There are studies with radiologic confirmation to predict the position of the end of the catheter in the epidural space, when the epidural catheter is inserted toward the cephalad and caudad needle directions [15-18]. Kawagoe et al. [16] conducted radiologic confirmation of epidural catheter insertion $7 \mathrm{~cm}$ toward the cephalad direction, when epidural anesthesia was carried out for a gynecology operation. Only $11.7 \%$ of catheterization was carried out along the intended direction, which was greater than the spinal segment toward the cephalad. Lim et al. [17] conducted radiologic confirmation of epidural catheter insertion toward the cephalad and caudad directions for epidural anesthesia, and the end of the catheter in the two directions was coiled at $2.9 \mathrm{~cm}$ and $2.5 \mathrm{~cm}$, respectively. They suggested the appropriate depth for the epidural catheter insertion to be $3 \mathrm{~cm}$, as only $13 \%$ of the catheter was progressed more than $4 \mathrm{~cm}$ without coiling. In addition, radiologic confirmation was carried out for epidural anesthesia for gynecologic operations, and the end of the catheter was positioned toward the caudad direction more frequently in the case of $7 \mathrm{~cm}$ insertion to cephalad than in $5 \mathrm{~cm}$ insertion to cephalad. The report concluded that there is a possibility of the catheter position being in the opposite direction, as the end of catheter is coiled with deeper 
insertion [18]. It is not clinically feasible to conduct radiologic confirmation of the epidural catheter position in every patient. The study selected $3 \mathrm{~cm}$ as the insertion depth for the epidural catheter to prevent the catheter from coiling and to match the direction of the catheter insertion and the catheter position.

In conclusion, in elderly patients under continuous epidural anesthesia for urological endoscopic surgery, there was no significant difference in the rate of paresthesia prevalence between the patients in whom the catheter was inserted toward the caudad direction, for sufficient anesthesia in the sacral area, and in whom it was inserted toward the cephalad direction; this finding has implications for the clinical practice.

\section{References}

1. Galindo A, Hernandez J, Benavides O, Ortegon de Munoz S, Bonica JJ. Quality of spinal extradural anaesthesia: the influence of spinal nerve root diameter. Br J Anaesth 1975; 47: 41-7.

2. Chou WY, Hsu CJ, Cheng JT, Yang LC, Lin CR, Chia YY, et al. Anesthetic effect of epidural anesthesia with cephalad or caudad catheterization for ankle surgery or hemorrhoidectomy. Acta Anaesthesiol Scand 2005; 49: 406-10.

3. Muñoz HR, Dagnino JA, Allende M, Bugedo GJ, Montes JM, Cherres OG. Direction of catheter insertion and incidence of paresthesias and failure rate in continuous epidural anesthesia: a comparison of cephalad and caudad catheter insertion. Reg Anesth 1993; 18: 331-4.

4. Bogduk N. The innervation of the lumbar spine. Spine (Phila Pa 1976) 1983; 8: 286-93.

5. Raoul S, Faure A, Robert R, Rogez JM, Hamel O, Cuillère P, et al. Role of the sinu-vertebral nerve in low back pain and anatomical basis of therapeutic implications. Sur Radiol Anat 2003; 24: 366-71.

6. Horlocker TT, McGregor DG, Matsushige DK, Schroeder DR, Besse JA. A retrospective review of 4767 consecutive spinal anesthetics: central nervous system complications. Perioperative Outcome Group. Anesth Analg 1997; 84: 578-84.

7. Aldrete JA. Neurologic deficits and arachnoiditis following neuroaxial anesthesia. Acta Anaesthesiol Scand 2003; 47: 3-12.

8. Rolbin SH, Hew E, Ogilvie G. A comparison of two types of epidural catheters. Can J Anaesth 1987; 34: 459-61.

9. Sarna MC, Smith I, James JM. Paraesthesia with lumbar epidural catheters: A comparison of air and saline in a loss-of-resistance technique. Anaesthesia 1990; 45: 1077-9.

10. Kim SY, Kim YY, Kim AR. Incidence of intravascular insertion in thoracic epidural catheterization by using real time fluoroscopy. Korean J Anesthesiol 2012; 62: 251-5.

11. Cesur M, Alici HA, Erdem AF, Silbir F, Yuksek MS. Administration of local anesthetic through the epidural needle before catheter insertion improves the quality of anesthesia and reduces catheterrelated complications. Anesth Analg 2005; 101: 1501-5.

12. Cartagena R, Gaiser RR. Advancing an epidural catheter $10 \mathrm{~cm}$ then retracting it $5 \mathrm{~cm}$ is no more effective than advancing it $5 \mathrm{~cm}$. J Clin Anesth 2005; 17: 528-30.

13. Ong BY, Cohen MM, Esmail A, Cumming M, Kozody R, Palahnuik RJ. Paresthesia and motor dysfunction after labour and delivery. Anesth Analg 1987; 66: 18-22.

14. Terasako K. Experience of lumbar epidural insertion in 573 anesthetized patients. J Anesth 2011; 25: 950-1.

15. Tiso RL, Thomas PS, Macadaeg K. Epidural catheter direction and local anesthetic dose. Reg Anesth 1993; 18: 308-11.

16. Kawagoe I, Tajima K, Kanai M, Mitsuhata H. Confirmation of the position of the epidural catheter regarding its intended direction and location for lumbar epidural block. Masui 2009; 58: 692-9.

17. Lim YJ, Bahk JH, Ahn WS, Lee SC. Coiling of lumbar epidural catheters. Acta Anaesthesiol Scand 2002; 46: 603-6.

18. Kawagoe I, Tajima K, Kanai M, Mitsuhata H. The direction and the position of epidural catheter tips inserted $5 \mathrm{~cm}$ or $7 \mathrm{~cm}$ cephalad did not differ. Masui 2010; 59: 1171-7. 